\title{
Neuropsychiatric Systemic Lupus Erythematosus: A Diagnostic Conundrum
}

\author{
Vivek Joseph ${ }^{\mathrm{a}}$, Rahul Anil ${ }^{\mathrm{b}, \mathrm{d}}$, Sary Aristy ${ }^{\mathrm{c}}$
}

\begin{abstract}
A 70-year-old man presented with complaints of rapid cognitive decline and new onset leukopenia. The patient had a 17-year history of refractory seizures. Detailed review of symptoms and investigations revealed the patient met American College of Rheumatology (ACR) diagnostic criteria for systemic lupus erythematosus (SLE). The patient had high titer ANA with a strongly positive dsDNA. Immunosuppressive therapy with hydroxychloroquine and mycophenolate mofetil led to significant improvement in cognition and seizures. Neuropsychiatric SLE should be considered a potential differential diagnosis for patients presenting with seizures or cognitive decline. Moreover, neuropsychiatric manifestations especially seizures are an early event in the disease course of SLE. Hence, we believe that early diagnosis of SLE by neuropsychiatric manifestations will not only lead to better control of CNS symptoms but early immunosuppressive therapy could control the progression of the underlying autoimmune disease.
\end{abstract}

Keywords: Neuropsychiatric manifestations; Seizures; Cognitive decline; Immunosuppressive therapy

\section{Introduction}

The hallmark of systemic lupus erythematosus (SLE) is its autoimmunity and protean clinical manifestations that can affect virtually every organ and can vary perilously living up to its name as "the disease with thousand faces". The extent of CNS manifestations can vary broadly, from less severe subclinical abnormalities in neurocognitive function, such as memory, intellect and learning to more grueling presentations,

Manuscript accepted for publication August 18, 2016

aDepartment of Internal Medicine, Rutgers New Jersey Medical School, Rutgers University, 150 Bergen Street, Newark, NJ 07601, USA

bepartment of Neurology, Yale School of Medicine, Yale University, 15 York Street, New Haven, CT 06510, USA

'Department of Rheumatology, Rheumatology Associates of North Jersey, 1415 Queen Anne Rd, Teaneck, NJ 07666, USA

${ }^{\mathrm{d} C}$ Corresponding Author: Rahul Anil, Department of Neurology, Yale School of Medicine, Yale University, 15 York Street, New Haven, CT 06510, USA. Email: drrahulanil@gmail.com

doi: http://dx.doi.org/10.14740/jocmr2698w such as seizure, stroke or transverse myelitis. The incidence of epilepsy among SLE patients increases three times more than the general population and patients with both SLE and epilepsy are more prone to experience neuropsychiatric disorders. The understanding of disparate pathogenic mechanisms and therapy involved are impecunious. We present a case of a 70 -year-old male who presented with a 17 -year history of epilepsy and cognitive decline diagnosed with SLE and subsequently responded well to immunotherapy.

\section{Case Report}

A 70-year-old Middle Eastern male with a past medical history of poorly controlled seizure disorder, HTN, and atrial fibrillation was admitted to the hospital due to worsening cognitive decline and leukopenia noticed on outpatient lab work. Patient was diagnosed with epilepsy 17 years ago and family members note it is poorly controlled on his current regimen of clobazam 5 and $10 \mathrm{mg}$, levetiracetam 1,000 and $1,500 \mathrm{mg}$, and lacosamide 200 and $250 \mathrm{mg}$ (morning and evening, respectively). His seizures were generalized, jerky movements of extremities, pronounced on the right side, with post-ictal confusion and incoherent speech.

Initial blood workup revealed significant leukopenia, proteinuria on urine analysis and bilateral hilar, mesenteric and inguinal lymphadenopathy on CT. Bone marrow biopsy was suggestive of myelodysplastic syndrome. Right inguinal node biopsy showed no evidence of malignancy. During the hospital course, patient developed swelling and tenderness of the bilateral wrist joints making us consider a rheumatologic etiology for his symptoms. On further questioning, the family noted that the patient frequently developed pain and swelling of the joints, predominantly in knee and hands. Rheumatological workup revealed a high ANA titer of 1:640, dsDNA strongly positive, $\mathrm{C} 3 / \mathrm{C} 4$ was low and $\mathrm{RF}$, anti-CCP was negative.

Patient was put on oral prednisone $60 \mathrm{mg}$ once daily resulting in improvement of joint swelling and cognition. Patient was discharged on oral prednisone and hydroxychloroquine $200 \mathrm{mg}$ twice daily with outpatient follow-up. As an outpatient, the benefits and disadvantage of renal biopsy to work up proteinuria were discussed with the family and finally it was decided not to perform renal biopsy due to high bleeding risk expected due to anticoagulation (warfarin), and the patient was put on mycophenolate mofetil (MMF) $100 \mathrm{mg}$ twice daily, for likely lupus nephritis. Eventually, steroids were gradually tapered off and patient was continued with hydroxychloroquine 
and MMF (200 and 1,000 mg twice daily, respectively) on outpatient course. Patient has been seizure free for the past 5 months of outpatient therapy. Patient's mental status has significantly improved and is now able to perform activities of daily living independently which he could not previously.

\section{Discussion}

SLE is a chronic, relapsing remitting systemic disease characterized by loss of immune tolerance resulting in autoantibody production with immune complex deposition. It has a predisposition towards females of a childbearing age with a female to male ratio that ranges from 7:1 to $15: 1$. Hence, this case of SLE diagnosed in a 70-year-old male shows the variable presentations of this disease and why it is rightly called the disease with a thousand faces.

The American College of Rheumatology (ACR) has designed specific diagnostic criteria requiring at least 4 out of 11 to yield a sensitivity of $85 \%$ and a specificity of $95 \%$ [1]. This fact is well validated in our case of a 70-year-old man with a score of 6 out of 11 projecting a diagnosis of SLE with even more greater statistical strength - arthritis, leukopenia, seizures, persistent proteinuria with positive ANA, and anti-dsDNA.

Neuropsychiatric manifestations of SLE have been divided by the ACR into cerebrovascular disease, cognitive dysfunction, seizures and psychosis, and peripheral nervous system disorders. The manifestation of this neuropsychiatric lupus can take place in absence of either serologic activity or other metabolic disorders [2]. Prevalence of neuropsychiatric manifestations of SLE ranges from $11 \%$ to $90 \%$ with large variation among cohort studies [3]. Review of the literature presents certain hypothesis of the pathogenesis of seizures and cognitive dysfunction in patients with SLE. Antiphospholipid antibody inhibiting Y-aminobutyric acid receptor-ion channel complex has been shown to increase neuronal excitability [4, 5]. Denburg and colleagues illustrated antiphospholipid antibody was associated with cognitive decline in certain aspects (verbal memory, cognitive flexibility, and psychomotor speed) [6]. However, it is important to note that antiphospholipid antibody was negative in this patient. Blood-brain barrier integrity dysfunction due to autoantibodies in SLE patients may also play a role in cognitive decline. Kowal et al demonstrated in animal model that anti-glutamate receptor antibodies are associated with cognitive dysfunction and hippocampal apoptosis in the presence of blood-brain barrier disruption $[7,8]$.

Incidence of epilepsy among SLE patients is three times more than the general population [9]. Patients with SLE and epilepsy are more likely to experience neuropsychiatric disorders. A cohort study by Huang et al showed 56 (26.3\%) out of 213 patients with a history of seizures had the first seizures more than 1 year before diagnosis of SLE [10]. Appenzeller et al illustrated the frequency and risk factors of epileptic seizures in a large cohort of patients with SLE which showed 19 of $60(31.6 \%)$ had epileptic seizure at the onset of SLE disease activity, 41 of $60(68 \%)$ had epileptic seizures after disease onset and seven of $60(11.7 \%)$ had recurrence [11]. Therefore, seizure can occur at any time either before, at, or after SLE diagnosis. Ramsey-Goldman et al stated in the study that seizures are a relatively early event in the disease course of SLE [12]. Hence, we believe that early diagnosis of SLE by neuropsychiatric manifestations will not only lead to better control of CNS symptoms but early immunosuppressive therapy could control the progression of the underlying autoimmune disease.

We postulate that the reduced frequency of confusional episodes reflecting subclinical seizures and significant improvement in cognitive function in our patient are attributable to the immunosuppressive effect of hydroxychloroquine and MMF. This is validated by Sommerlad et al who concluded immuno-modulating therapy significantly improves both subjective and objective clinical presentations of neuropsychiatric SLE [13]. Considering toxicity profile current view favors MMF to cyclophosphamide, which has much better side effect profile for maintenance and initial treatment of neuropsychiatric lupus. Also, MMF demonstrated a significantly higher remission rate than intravenous cyclophosphamide in renal lupus in the randomized, open label, non-inferiority trial conducted by Ginzler et al [14]. Above evidence convinced us to start the patient on MMF.

\section{Conclusion}

In conclusion, this case highlights the fact that seizures and other neuropsychiatric manifestations of SLE can often predate other symptoms making the diagnosis of CNS lupus a challenging one due to the lack of specific laboratory tests, non-specific neuroimaging findings, and inaccessible brain parenchyma for histological confirmation and overall non-specific nature of the disease. We also believe that early diagnosis and initiation of immunosuppressive therapy will not only lead to better control of CNS symptoms but early immunosuppressive therapy could defer the progression of the underlying autoimmune disease.

\section{Conflicts of Interest}

All authors have no conflicts of interest or financial gain.

\section{Author Contributions}

All authors contributed the same.

\section{References}

1. Hochberg MC. Updating the American College of Rheumatology revised criteria for the classification of systemic lupus erythematosus. Arthritis Rheum. 1997;40(9):1725.

2. Harel L, Sandborg C, Lee T, von Scheven E. Neuropsychiatric manifestations in pediatric systemic lupus erythematosus and association with antiphospholipid antibodies. J Rheumatol. 2006;33(9):1873-1877.

3. Benseler SM, Silverman ED. Neuropsychiatric involve- 
ment in pediatric systemic lupus erythematosus. Lupus. 2007;16(8):564-571.

4. Liou HH, Wang CR, Chou HC, Arvanov VL, Chen RC, Chang YC, Chuang CY, et al. Anticardiolipin antisera from lupus patients with seizures reduce a GABA receptor-mediated chloride current in snail neurons. Life Sci. 1994;54(15):1119-1125.

5. Chapman J, Cohen-Armon M, Shoenfeld Y, Korczyn AD. Antiphospholipid antibodies permeabilize and depolarize brain synaptoneurosomes. Lupus. 1999;8(2):127-133.

6. Denburg SD, Carbotte RM, Ginsberg JS, Denburg JA. The relationship of antiphospholipid antibodies to cognitive function in patients with systemic lupus erythematosus. J Int Neuropsychol Soc. 1997;3(4):377-386.

7. Kowal C, Degiorgio LA, Lee JY, Edgar MA, Huerta PT, Volpe BT, Diamond B. Human lupus autoantibodies against NMDA receptors mediate cognitive impairment. Proc Natl Acad Sci U S A. 2006;103(52):19854-19859.

8. Omdal R, Brokstad K, Waterloo K, Koldingsnes W, Jonsson R, Mellgren SI. Neuropsychiatric disturbances in SLE are associated with antibodies against NMDA receptors. Eur J Neurol. 2005;12(5):392-398.
9. Unterman A, Nolte JE, Boaz M, Abady M, Shoenfeld Y, Zandman-Goddard G. Neuropsychiatric syndromes in systemic lupus erythematosus: a meta-analysis. Semin Arthritis Rheum. 2011;41(1):1-11.

10. Huang X, Magder LS, Petri M. Predictors of Incident Seizure in Systemic Lupus Erythematosus. J Rheumatol. 2016;43(3):565-575.

11. Appenzeller S, Cendes F, Costallat LT. Epileptic seizures in systemic lupus erythematosus. Neurology. 2004;63(10):1808-1812.

12. Ramsey-Goldman R, Alarcon GS, McGwin G, Petri M, Vila LM, Edberg JC, Reveille JD, et al. Time to seizure occurrence and damage in PROFILE, a multiethnic systemic lupus erythematosus cohort. Lupus. 2008;17(3):177-184.

13. Sommerlad A, Duncan J, Lunn MP, Foong J. Neuropsychiatric systemic lupus erythematosus: a diagnostic challenge. BMJ Case Rep. 2015;2015.

14. Ginzler EM, Dooley MA, Aranow C, Kim MY, Buyon J, Merrill JT, Petri M, et al. Mycophenolate mofetil or intravenous cyclophosphamide for lupus nephritis. N Engl J Med. 2005;353(21):2219-2228. 